\title{
Constraints on cosmological models from strong gravitational lensing systems
}

\author{
Shuo Cao ${ }^{1}$, Yu Pan ${ }^{1,2}$, Marek Biesiada ${ }^{3}$, Wlodzimierz Godlowski ${ }^{4}$ and Zong-Hong Zhu ${ }^{1}$ \\ 1 Department of Astronomy, Beijing Normal University, Beijing 100875, China; \\ zhuzh@bnu.edu.cn \\ ${ }^{2}$ College Mathematics and Physics, Chongqing Universe of Posts and Telecommunications, \\ Chongqing 400065, China \\ 3 Department of Astrophysics and Cosmology, Institute of Physics, University of Silesia, \\ Uniwersytecka 4, 40-007 Katowice, Poland \\ ${ }^{4}$ Institute of Physics, Opole University, Oleska 48, 45-052 Opole, Poland
}

\begin{abstract}
Strong lensing has developed into an important astrophysical tool for probing both cosmology and galaxies (their structure, formation, and evolution). Using the gravitational lensing theory and cluster mass distribution model, we try to collect a relatively complete observational data concerning the Hubble constant independent ratio between two angular diameter distances $D_{d s} / D_{s}$ from various large systematic gravitational lens surveys and lensing by galaxy clusters combined with X-ray observations, and check the possibility to use it in the future as complementary to other cosmological probes. On one hand, strongly gravitationally lensed quasar-galaxy systems create such a new opportunity by combining stellar kinematics (central velocity dispersion measurements) with lensing geometry (Einstein radius determination from position of images). We apply such a method to a combined gravitational lens data set including 70 data points from Sloan Lens ACS (SLACS) and Lens Structure and Dynamics survey (LSD). On the other hand, a new sample of 10 lensing galaxy clusters with redshifts ranging from 0.1 to 0.6 carefully selected from strong gravitational lensing systems with both X-ray satellite observations and optical giant luminous arcs, is also used to constrain three dark energy models $(\Lambda \mathrm{CDM}$, constant $w$ and $\mathrm{CPL})$ under a flat universe assumption. For the full sample $(n=80)$ and the restricted sample ( $n=46)$ including 36 two-image lenses and 10 strong lensing arcs, we obtain relatively good fitting values of basic cosmological parameters, which generally agree with the results already known in the literature. This results encourages further development of this method and its use on larger samples obtained in the future.
\end{abstract}


Subject headings: Gravitational lensing: strong - (Cosmology:) cosmological parameters - (Cosmology:) dark energy

\section{Introduction}

Pioneering observations of type Ia supernovae (SNe Ia) (Riess et al. 1998; Perlmutter et al. 1999) have demonstrated that our present universe is passing through an accelerated phase of expansion preceded by a period of deceleration. A new type of matter with negative pressure known as dark energy, has come up to explain the present phase of acceleration. The simplest candidate of dark energy, the cosmological constant $(\Lambda)$, is consistent with various observations such as more precise supernova data (Riess et al. 2004; Davis et al. 2007; Kowalski et al. 2008), the CMB observations (Spergel et al. 2007; Komatsu et al. 2009), the light elements abundance from Big Bang Nucleosynthesis (Burles et al. 2001), the baryon acoustic oscillations (BAO) detected in SDSS sky survey (Eisenstein et al. 2005), radio galaxies (Daly et al. 2009), and gamma-ray bursts (Amati et al. 2008). However, various other models were proposed as candidates of dark energy, such as the typical dynamical scalar field called quintessence (Caldwell et al. 1998), phantom corrections (Caldwell 2002), a joint quintom scenario (Feng et al. 2005) or Chaplygin gas (Kamenshchik et al. 2001; Zhu 2004; Biesiada, Godlowski \& Szydlowski 2005; Zhang \&Zhu 2006), to mention just a few out of a long list. On the other hand there are still many other ways to understand the accelerating universe, such as Modified Friedmann Equation (Freese \& Lewis 2002; Zhu et al. 2004) and Dvali-Gabadadze-Porrati(DGP) mechanism (Dvali et al. 2000). But until now none of these models was demonstrated superior over the other. Besides, while updating the current estimates of cosmological model parameters, one should try to use new probes. Strongly gravitationally lensed systems belong to this category. They can provide the information on two angular diameter distances, $D_{d s}$ and $D_{s}$. One is the distance to the source and the other is that between the defector and the source. Since angular diameter distance depends on cosmological geometry, we can use their ratios to constrain cosmological models.

The discovery of strong gravitational lensing in Q0957+561 (Walsh et al. 1979) opened up an interesting possibility to use strong lens systems in the study of cosmology and astrophysics. Up to now, strong lensing has developed into an important astrophysical tool for probing both cosmology (Zhu 2000a, b; Chae 2003; Chae et al. 2004; Mitchell et al. 2005; Zhu \& Mauro 2008a; Zhu et al. 2008b) and galaxies (their structure, formation, and evolution) (Zhu \& Wu 1997; Mao \& Schneider 1998; Jin et al. 2000; Keeton 2001; Kochanek \& White 2001; Ofek et al. 2003; Treu et al. 2006a). Now several hundreds of strong lens systems pro- 
duced by massive galaxies have been discovered, but only $\sim 90$ galactic-scale strong lenses with known redshift of the lens and the source and measured image separation can form well-defined samples useful for statistical analyses. These well-defined strong lenses are particularly useful not only for constraining the statistical properties of galaxies such as stellar velocity dispersions or galaxy evolution (Chae \& Mao 2003; Ofek et al. 2003), but also for constraining cosmological parameters such as the present-day matter density $\Omega_{m}$, dark energy density $\Omega_{x}$ and its equation of state $w$ (Chae 2003; Mitchell et al. 2005). For example, the Cosmic Lens All-Sky Survey (CLASS) statistical data, which consists of 8958 radio sources out of which 13 sources are multiply imaged (Browne et al. 2003; Chae 2003) was first extensively used by Chae (2003), who found $\Omega_{m} \approx 0.3$ assuming a flat cosmology and non-evolving galaxy populations. Mitchell et al. (2005) reused this CLASS statistical sample based on the velocity dispersion function (VDF) of early-type galaxies derived from the SDSS Data Release 1 (DR1; Stoughton et al. (2002)). Zhu \& Mauro (2008a) reanalyzed 10 CLASS multiply-imaged sources whose image-splittings are known to be caused by single early-type galaxies to check the validity of the DGP model with radio-selected gravitational lensing statistics. More recently, the distribution of gravitationally-lensed image separations observed in the Cosmic Lens All-Sky Survey (CLASS), the PMN-NVSS Extragalactic Lens Survey (PANELS), the Sloan Digital Sky Survey (SDSS) and other surveys was used by Cao \& Zhu (2011a), who found $w<-0.52$ assuming a flat cosmology and adopting semianalytical modeling of galaxy formation. The idea of using strongly gravitationally lensed systems, in particular measurements of their Einstein radii combined with spectroscopic data, for measuring cosmological parameters including the cosmic equation of state was discussed in Biesiada (2006) and also in a more recent papers (Grillo et al. 2008; Biesiada et al. 2010; Biesiada, Malec \& Piórkowska 2011).

On the other hand, galaxy clusters, as the largest dynamical structures in the universe, are also widely used both in cosmology and astrophysics. Firstly, their mass distributions at different redshifts can be described by the Press-Schechter function (Press \& Schechter 1974), which reflects the linear growth rate of density perturbations and therefore can provide constraints on cosmological parameters such as the matter and dark energy densities (Borgani et al. 1999). Secondly, combining the Sunyaev-Zel'dovich effect (Sunvaev \& Zeldovich 1972) with observations of clusters' X-ray luminosity, one is able to measure or estimate the Hubble constant and other cosmological parameters in given cosmological model (Reese et al. 2002; Schmidt et al. 2004; Jones et al. 2005; Bonamente et al. 2006; Zhu \& Fujimoto 2004). Relevant discussions on the corrections to the Sunyaev-Zeldovich effect for galaxy clusters can be found in Itoh et al. (1998); Nozawa et al. (1998, 2006). More importantly, giant arcs generated by the galaxy cluster are perfect indicators of its surface mass density, while the mass distribution of the cluster's mass halo can be modelled from X-ray luminosity and 
temperature, which may provide certain observable (Sereno 2002; Sereno \& Longo 2004). Recently, Yu \& Zhu (2010) collected a new sample with such data from an online database BAX and various literature, which led to some interesting results compared with those obtained by Sereno \& Longo (2004).

In this paper, we try to collect a relatively complete observational data concerning the Hubble constant independent ratio between two angular diameter distances $D_{d s} / D_{s}$ from various large systematic gravitational lens surveys and galaxy cluster data. This paper is organized as follows. In Section 2, we briefly describe the methodology for both strong gravitationally lensed systems: galactic lenses and galaxy clusters. Then, in Section 3 we present the $D_{d s} / D_{s}$ data from various large systematic gravitational lens surveys and lensing galaxy clusters with X-ray observations and optical giant luminous arcs. We further introduce three popular cosmological models tested in Section 4. Finally, we show the results of constraining cosmological parameters using MCMC method and conclude in Section 5 .

\section{The Method}

Gravitational lensing is one of the successful predictions of General Relativity. Strong gravitational lensing occurs whenever the source, the lens and the observer are so well aligned that the observer-source direction lies inside the so-called Einstein ring of the lens. Paczynski \& Gorski (1981) tried to use lensing images as indicators to estimate cluster mass and constrain cosmological constant.

In a cosmological context the source is usually a quasar with a galaxy acting as the lens. Strong lensing reveals itself as multiple images of the source, and the image separations in the system depend on angular diameter distances to the lens and to the source, which in turn are determined by background cosmology. Since the discovery of the first gravitational lens the number of strongly lensed systems increased to a hundred (in the CASTLES data base) and is steadily increasing following new surveys like the Sloan Lens ACS (SLACS) survey (Newton et al. 2011). This opens a possibility to constraining the cosmological model provided that we have good knowledge of the lens model.

Now, the idea is that the formula for the Einstein radius in a SIS lens (or its SIE equivalent),

$$
\theta_{E}=4 \pi \frac{D_{A}\left(z, z_{s}\right)}{D_{A}\left(0, z_{s}\right)} \frac{\sigma_{S I S}^{2}}{c^{2}}
$$

depends on the cosmological model through the ratio of (angular diameter) distances between lens and source and between observer and lens. Under flat Friedman-Walker metric, the 
angular diameter distance reads

$$
D_{A}(z ; \mathbf{p})=\frac{c}{H_{0}(1+z)} \int_{0}^{z} \frac{d z^{\prime}}{E\left(z^{\prime} ; p\right)} .
$$

where $H_{0}$ is the Hubble constant and $E(z ; \mathbf{p})$ is a dimensionless expansion rate dependent on redshift $z$ and cosmological model parameters $\mathbf{p}$. If the Einstein radius $\theta_{E}$ from image astrometry and stellar velocity dispersion $\sigma$ (or central velocity dispersion $\sigma_{0}$ ) from spectroscopy can be determined, this method can be used to constrain cosmological parameters. The advantage of this method is that it is independent of the Hubble constant value and is not affected by dust absorption or source evolutions. However, it depends on the measurements of $\sigma_{0}$ and lens modelling (e.g. singular isothermal sphere (SIS) or singular isothermal ellipsoid (SIE) assumption). Hopefully, spectroscopic data for central parts of lens galaxies became available from the Lens Structure and Dynamics (LSD) survey and the more recent SLACS survey etc, which make it possible to assess the central velocity dispersions $\sigma_{0}$ (Treu et al. 2006a,b; Grillo et al. 2008). Meanwhile, the SIS (or SIE) model is still a useful assumption in gravitational lensing studies and should be accurate enough as first-order approximation to the mean properties of galaxies relevant to statistical lensing. For example, Koopmans et al. (2009) found that inside one effective radius massive elliptical galaxies are kinematically indistinguishable from an isothermal ellipsoid. In the previous works, such an isothermal mass profile has also been widely used for analyses of statistical lensing (Kochanek 1996; King et al. 1997; Fassnacht \& Cohen 1998; Rusin \& Kochanek 2005; Koopmans et al. 2006, 2009; Treu et al. 2006a, b).

However, let us note here that the velocity dispersion $\sigma_{S I S}$ of the mass distribution and the observed stellar velocity dispersion $\sigma_{0}$ need not be the same. White \& Davis (1996) argued that there is a strong indication that dark matter halos are dynamically hotter than the luminous stars based on X-ray observations, and dark matter must necessarily have a greater velocity dispersion than the visible stars. In this paper, we adopt a free parameter $f_{E}$ that relates the velocity dispersion $\sigma_{S I S}$ and the stellar velocity dispersion $\sigma_{0}$ (Kochanek 1992; Ofek et al. 2003):

$$
\sigma_{S I S}=f_{E} \sigma_{0}
$$

To be more specific, we have kept $f_{E}$ as a free parameter, since it mimics the effects of: (i) systematic errors in the rms difference between $\sigma_{0}$ (observed stellar velocity dispersion) and $\sigma_{S I S}$ (SIS model velocity dispersion); (ii) the rms error caused by assuming the SIS model in order to translate the observed image separation into $\theta_{E}$, since the observed image separation does not directly correspond to $\theta_{E}$; (iii) softened isothermal sphere potentials which tend to decrease the typical image separations (Naravan \& Bartelmann 1996), and could be represented by $f_{E}$ somewhat smaller than 1 . 
Martel et al. (2002) found that the presence of the background matter tends to increase the image separations produced by lensing galaxies from ray-tracing simulations in CDM models, though this effect is small, of order of $20 \%$ or less. Christlein (2000) showed that richer environments of early type galaxies may have a higher ratio of dwarf to giant galaxies than the field. However, Keeton et al. (2000) showed that this effect nearly cancels the effect of the background matter, making the distribution of image separations significantly independent of environment. They predicted that lenses in groups have a mean image separation which is $\sim 0.2^{\prime \prime}$ smaller than that of lenses in the field. Therefore, all these above factors can possibly affect the images separation by up to $\pm 20 \%$, which may be mimicked by introducing $(0.8)^{1 / 2}<f_{E}<(1.2)^{1 / 2}$ (Ofek et al. 2003).

In the method we use, the cosmological model enters not through a distance measure directly, but rather through a distance ratio

$$
\mathcal{D}^{\text {th }}\left(z_{d}, z_{s} ; \mathbf{p}\right)=\frac{D_{d s}}{D_{s}}=\frac{\int_{z_{d}}^{z_{s}}\left[d z^{\prime} / E\left(z^{\prime} ; \mathbf{p}\right)\right]}{\int_{0}^{z_{s}}\left[d z^{\prime} / E\left(z^{\prime} ; \mathbf{p}\right)\right]}
$$

and respective observable counterpart reads

$$
\mathcal{D}^{o b s}=\frac{c^{2} \theta_{E}}{4 \pi \sigma_{0}^{2} f_{E}^{2}}
$$

with its corresponding uncertainty calculated through propagation equation concerning the errors both on the stellar velocity dispersion $\sigma_{0}$ and the Einstein radius $\theta_{E}(\sim 5 \%$ error for the Einstein radius (Grillo et al. 2008)).

Another source of systematic errors in our method comes from the fact that Einstein radius estimation from observed image positions depends on the lens model (SIS or SIE or the other realistic mass distribution). Moreover, the image separation could be affected by nearby masses (satellites, neighbor galaxies) or the structures along the line of sight. This last issue will also be discussed in the last section. Here, let us note that formally, at the level of Eq.(5) the $f_{E}$ factor does the double job accounting for systematics associated both with $\sigma_{0}$ and $\theta_{E}$. Since the main goal of this paper is to constrain cosmological parameters, we firstly consider $f_{E}$ as a free parameter, obtain its best-fit value and probability distribution function $P\left(f_{E}\right)$, and then treat it as a "nuisance" parameter to determine constraints on the relevant cosmological parameters of interest. The procedure of marginalization is carried out following that of Allen et al. (2008); Samushia \& Ratra (2008), where $P\left(f_{E}\right)$ is normalized to one and is usually taken to be a Gaussian or a $\delta\left(f_{E}\right)$ function peaked at the best-fit value of $f_{E}^{*}$. We then integrate the likelihood function,

$$
\mathcal{L}(\mathbf{p})=\int \mathcal{L}\left(\mathbf{p}, f_{E}\right) P\left(f_{E}\right) d f_{E}
$$


and determine the best-fit values and confidence level contours from $\mathcal{L}(\mathbf{p})$.

Moreover, strong lensing by clusters with galaxies acting as sources can produces giant arcs around galaxy clusters, which can also be used to constrain clusters' projected mass and cosmological parameters (Lynds \& Petrosian 1986; Breimer \& Sanders 1992; Sereno \& Longo 2004). When a galaxy cluster is relaxed enough, the hydrostatic isothermal spherical symmetric $\beta$-model (Cavaliere \& Fusco-Femiano 1976) can be used to describe the intracluster medium(ICM) density profile: $n_{e}(r)=n_{e 0}\left(1+r^{2} / r_{c}^{2}\right)^{-3 \beta_{X} / 2}$, where $n_{e 0}$ is the central electron density, $\beta_{X}$ and $r_{c}$ denote the slope and the core radius, respectively. Assuming that whole gas volume is isothermal (with the temperature $T_{X}$ ), the gravity of relaxed cluster and its gas pressure should balance each other according to the hydrostatic equilibrium condition. With the approximation of spherical symmetry, the cluster mass profile can be given by $M(r)=\frac{3 k_{B} T_{X} \beta_{X}}{G \mu m_{p}} \frac{r^{3}}{r_{c}^{2}+r^{2}}$, where $k_{B}, m_{p}$ and $\mu=0.6$ are, respectively, the Boltzmann constant, the proton mass, and the mean molecular weight (Rosati et al. 2002). A theoretical surface density can be derived as

$$
\Sigma_{t h}=\frac{3}{2 G \mu m_{p}} \frac{k_{B} T_{X} \beta_{X}}{\theta_{c}} \frac{1}{D_{d}} .
$$

Combining this with the critical surface mass density for lensing arcs (Schneider et al. 1992)

$$
\Sigma_{o b s}=\frac{c^{2}}{4 \pi G} \frac{D_{s}}{D_{d} D_{d s}} \sqrt{\frac{\theta_{t}^{2}}{\theta_{c}^{2}}+1}
$$

a Hubble constant independent ratio can be obtained

$$
\mathcal{D}^{o b s}=\left.\frac{D_{d s}}{D_{s}}\right|_{o b s}=\frac{\mu m_{p} c^{2}}{6 \pi} \frac{1}{k_{B} T_{X} \beta_{X}} \sqrt{\theta_{t}^{2}+\theta_{c}^{2}}
$$

The X-ray data fitting results may provide us the above mentioned relevant parameters such as $T_{X}, \beta_{X}$, and $\theta_{c}$. The position of tangential critical curve $\theta_{t}$ is usually deemed to be equal to the observational arc position $\theta_{\text {arc }}$. In this paper we assume that the deflecting angle has a slight difference with the arc radius angle, $\theta_{t}=\epsilon \theta_{\text {arc }}$, with the correction factor $\epsilon=(1 / \sqrt{1.2}) \pm 0.04$ (Ono et al. 1999). The complete set of standard priors and allowances of the above parameters included in Eq. [9] can be found in Table 1 of $Y u$ \& Zhu (2010). The observational $\mathcal{D}^{\text {obs }}$ and its corresponding uncertainty are also calculated through Eq. [9].

We stress here that the observational distance ratio $\mathcal{D}$ has both advantage and disadvantage. The positive side is that the Hubble constant $H_{0}$ gets cancelled, hence it does not introduce any uncertainty to the results. The disadvantage is that the power of estimating $\Omega_{m}$ is relatively poor (Biesiada et al. 2010). Therefore we only attempt to fit $\Omega_{m}$ in the 
case of a $\Lambda \mathrm{CDM}$ model (where it is the only free parameter in flat cosmology). Then for both cases above (Eq. [5 and Eq. 9]), we can fit theoretical models to observational data by minimizing the $\chi^{2}$ function

$$
\chi^{2}(\mathbf{p})=\sum_{i} \frac{\left(\mathcal{D}_{i}^{t h}(\mathbf{p})-\mathcal{D}_{i}^{o b s}\right)^{2}}{\sigma_{\mathcal{D}, i}^{2}} .
$$

where the sum is over the sample and $\sigma_{\mathcal{D}, i}^{2}$ denotes the variance of $\mathcal{D}_{i}^{\text {obs }}$.

\section{Sample used}

For the Einstein ring data, we first use a combined sample of 70 strong lensing systems with good spectroscopic measurements of central dispersions from the SLACS and LSD surveys (Biesiada et al. 2010; Newton et al. 2011). Original data concerning the sample can be found in (Koopmans \& Treu 2002, 2003; Treu \& Koopmans 2004; Treu et al. 2006a) (see for details). In our sample of 70 lenses some have 2 images and some have 4 . There are some general arguments in favor of SIS model, but strictly speaking SIS lens should have only 2 images (Biesiada et al. 2010; Biesiada, Malec \& Piórkowska 2011), so one can try to use only 2 image systems out of the full sample. Therefore we selected a subsample of $n=36$ lenses, which is summarized in Table 1 where the names of lenses in the restricted sample are given in bold.

As for the strong lensing arcs, redshifts and temperatures of the galaxy clusters are always searched out directly from online databases, such as CDS (The Strasbourg astronomical Data Center) or NED (NASA/IPAC Extragalactic Database). Yu \& Zhu (2010) have chosen a new database established especially for X-ray galaxy clusters - BAX, which provides detailed information including $\beta$ and $\theta_{c}$. They also used the fitting results of Chandra, ROSAT, ASCA satellites and VIMOS-IFU survey (Ota \& Mitsuda 2004; Bonamente et al. 2006; Covone et al. 2005; Richard et al. 2007). The final statistical sample satisfy the following well-defined selection criteria. Firstly, the distance between the lens and the source should be always smaller than that between the arc source and the observer, $D_{d s} / D_{s}<1$, which rules out half of selected lensing arcs. Secondly, the arcs whose positions are too far from characteristic radius $\left(\theta_{\text {arc }}>3 \theta_{c}\right)$ should also be discarded (Yu \& Zhu 2010). At last Yu \& Zhu (2010) obtained a sample of 10 giant arcs with all necessary parameters listed in Table 1.

Now the observational $D_{d s} / D_{s}$ data containing 80 data points for cosmological fitting are summarized in Table 1, with errors calculated with error propagation equation. We also list a restricted sample containing 46 data points, which consists 36 two-image lenses and 10 
strong lensing arcs.

\section{Cosmological models tested}

All cosmological models we will consider in this paper are currently viable candidates to explain the observed acceleration. Given the current status of cosmological observations, there is no strong reason to go beyond the simple, standard cosmological model with zero curvature and cosmological constant $\Lambda$ (except for the conceptual problems arising when one attempts to reconcile its observed value with some estimate derived from fundamental arguments (Weinberg 1989)). However, it is still interesting to investigate alternative models. And we hope that future observations of more accurate $D_{d s} / D_{s}$ data could allow to better discriminate various competing candidates. In the MCMC simulations we assume for each class of models the best fit values of parameters found in the present work, and vary them within their $2 \sigma$ uncertainties. We assume spatial flatness of the Universe throughout the paper, since it is strongly supported by independent and precise experiments e.g. a combined 5-yr Wilkinson Microwave Anisotropy Probe (WMAP5), baryon acoustic oscillations (BAO) and supernova data analysis gives $\Omega_{t o t}=1.0050_{-0.0061}^{+0.0060}$ (Hinshaw et al. 2009). Moreover, the $\Omega_{m}=0.27$ prior is used except in the $\Lambda$ CDM model where the fit is attempted.

For comparison we also performed fits to the newly released Union2 SNe Ia data $(\mathrm{n}=557$

supernovae) from the Supernova Cosmology project covering a redshift range $0.015 \leq z \leq 1.4$ (Amanullah et al. 2010). In the calculation of the likelihood from $\mathrm{SNe}$ Ia, we marginalize over the nuisance parameter (Di Pietro \& Claeskens 2003)

$$
\chi_{\mathrm{SNe}}^{2}=A-\frac{B^{2}}{C}+\ln \left(\frac{C}{2 \pi}\right),
$$

where $A=\sum_{i}^{557}\left(\mu^{\text {data }}-\mu^{\text {th }}\right)^{2} / \sigma_{i}^{2}, B=\sum_{i}^{557}\left(\mu^{\text {data }}-\mu^{\text {th }}\right) / \sigma_{i}^{2}, C=\sum_{i}^{557} 1 / \sigma_{i}^{2}$, and the distance modulus is $\mu=5 \log \left(d_{L} / \mathrm{Mpc}\right)+25$, with the $1 \sigma$ uncertainty $\sigma_{i}$ from the observations of SNe Ia; and the luminosity distance $d_{L}$ as a function of redshift $z$

$$
d_{L}=(1+z) \int_{0}^{z} \frac{c d z^{\prime}}{H_{0} E\left(z^{\prime} ; \mathbf{p}\right)}
$$

\subsection{The standard cosmological model $(\Lambda \mathrm{CDM})$}

We start our analysis by first setting out the predictions for the current standard cosmological model. In the simplest scenario, the dark energy is simply a cosmological constant, 
$\Lambda$, i.e. a component with constant equation of state $w=p / \rho=-1$. If flatness of the FRW metric is assumed, the Hubble parameter according to the Friedmann equation is

$$
E^{2}(z ; \mathbf{p})=\Omega_{m}(1+z)^{3}+\Omega_{\Lambda}
$$

where $\Omega_{m}$ and $\Omega_{\Lambda}$ parameterize the density of matter and cosmological constant, respectively. Moreover, in the zero-curvature case $\left(\Omega=\Omega_{m}+\Omega_{\Lambda}=1\right)$, this model has only one independent parameter: $\mathbf{p}=\Omega_{m}$.

\subsection{Dark energy with constant equation of state (wCDM)}

Allowing for a deviation from the simple $w=-1$ case, the accelerated expansion is obtained when $w<-1 / 3$. In a zero-curvature universe, the Hubble parameter for this generic dark energy component with density $\Omega_{x}$ then becomes

$$
E^{2}(z ; \mathbf{p})=\Omega_{m}(1+z)^{3}+\Omega_{x}(1+z)^{3(1+w)} .
$$

Obviously, when flatness and $\Omega_{m}=0.27$ are assumed, it is a one-parameter model with the model parameter: $\mathbf{p}=\{w\}$.

\subsection{Dark energy with variable equation of state (CPL)}

If the equation of state of dark energy is allowed to vary with time, one has to choose a suitable functional form for $w(z)$, which in general involves certain parametrization. Now, we consider the commonly used CPL model (Chevalier \& Polarski 2001; Linder 2003), in

which the equation of state of dark energy is parameterized as $w(z)=w_{0}+w_{a} \frac{z}{1+z}$, where $w_{0}$ and $w_{a}$ are constants. The corresponding $E(z)$ can be expressed as

$$
E^{2}(z ; \mathbf{p})=\Omega_{m}(1+z)^{3}+\left(1-\Omega_{m}\right)(1+z)^{3\left(1+w_{0}+w_{a}\right)} \exp \left(-\frac{3 w_{a} z}{1+z}\right) .
$$

There are two independent model parameters in this model: $\mathbf{p}=\left\{w_{0}, w_{a}\right\}$.

\section{Results and conclusions}

In the first case, we consider $f_{E}$ as a free parameter and show the constraint results with the full $n=70$ and the restricted $n=36$ two-image galaxy lenses in Fig. 1 and Fig. 2, In order to derive the probability distribution function for the cosmological parameters of interest, 

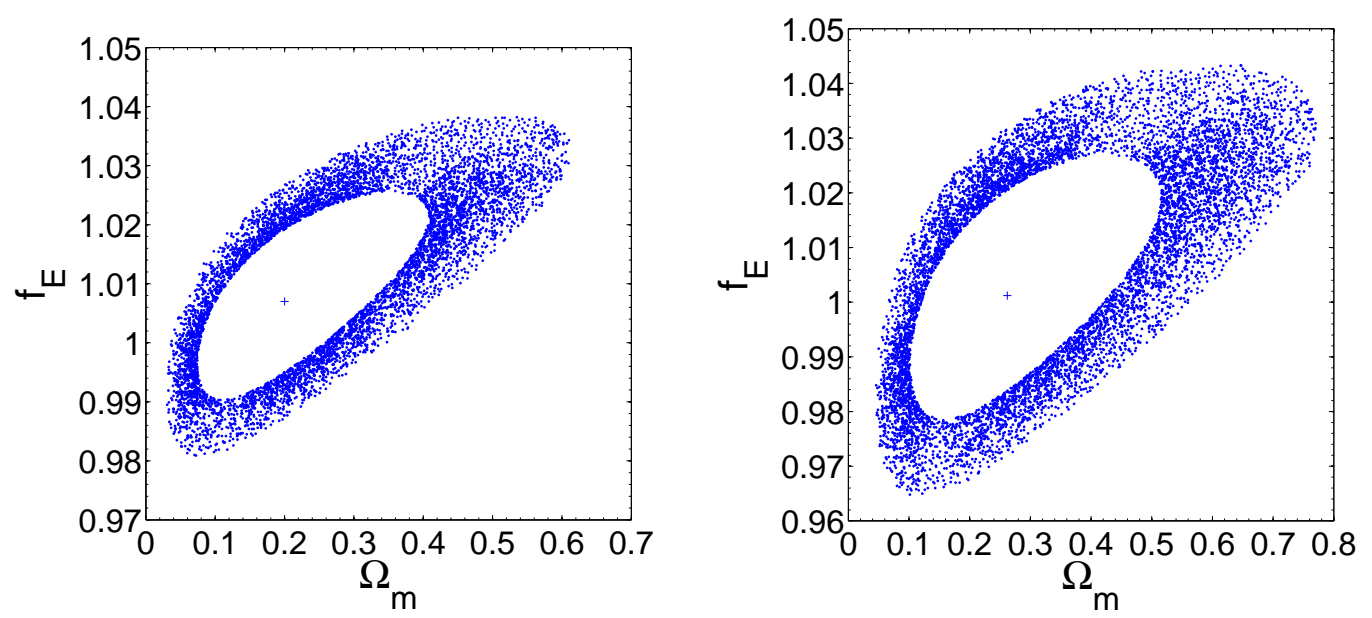

Fig. 1. - The 68.3 and $95.8 \%$ confidence regions for $\Lambda$ CDM model in the $\left(\Omega_{m}, f_{E}\right)$ plane obtained from the full $n=70$ and the restricted $n=36$ two-image galaxy lenses. The crosses represent the best-fit points.
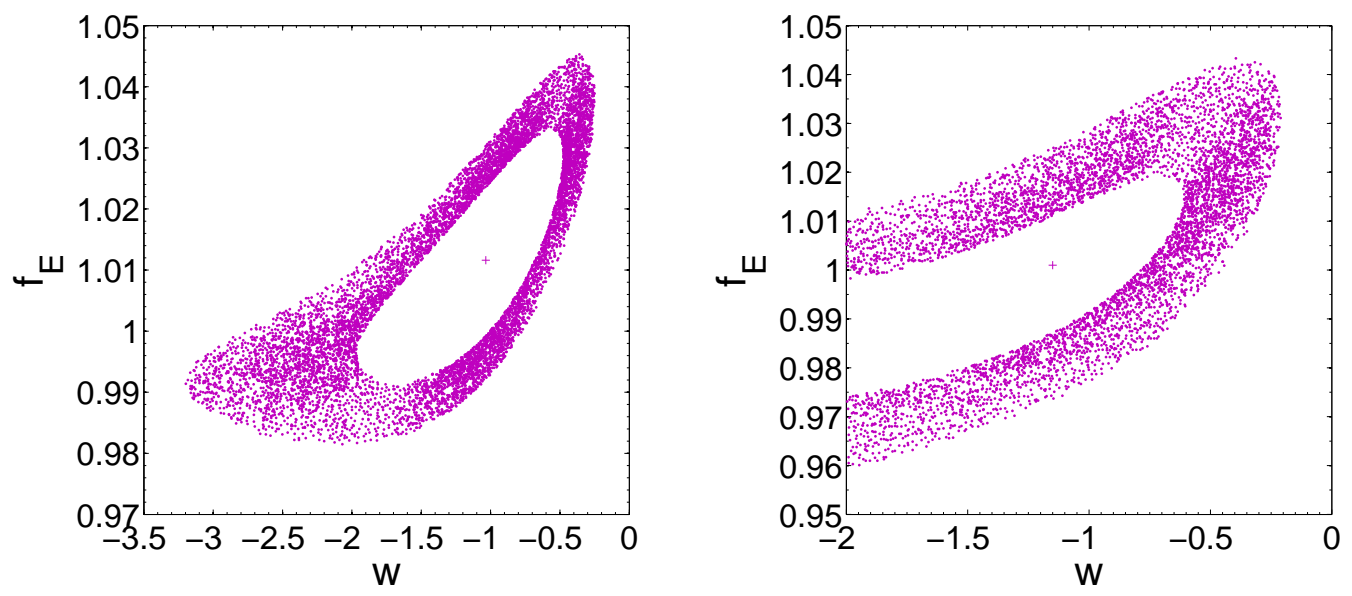

Fig. 2.- The 68.3 and $95.8 \%$ confidence regions for $w$ CDM model in the $\left(\mathrm{w}, f_{E}\right)$ plane obtained from the full $n=70$ and the restricted $n=36$ two-image galaxy lenses. The crosses represent the best-fit points. 

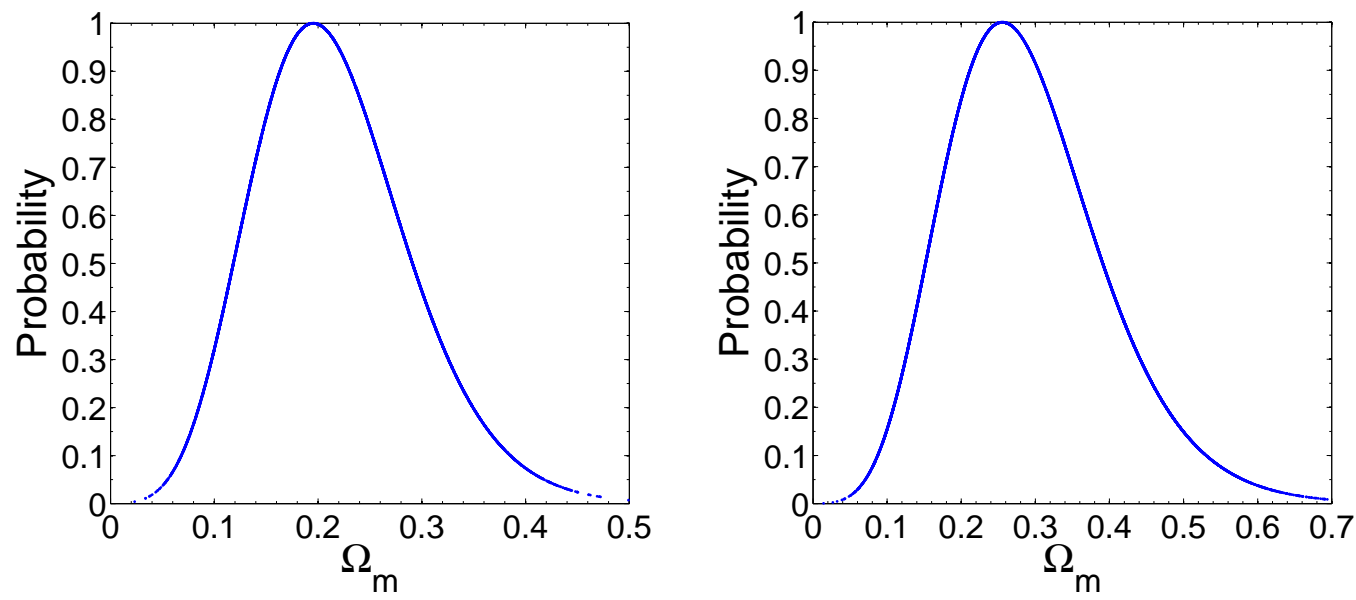

Fig. 3.- The marginalized constraint on $\Omega_{m}$ of $\Lambda$ CDM model from 80 full $D_{d s} / D_{s}$ data and 46 restricted $D_{d s} / D_{s}$ data.
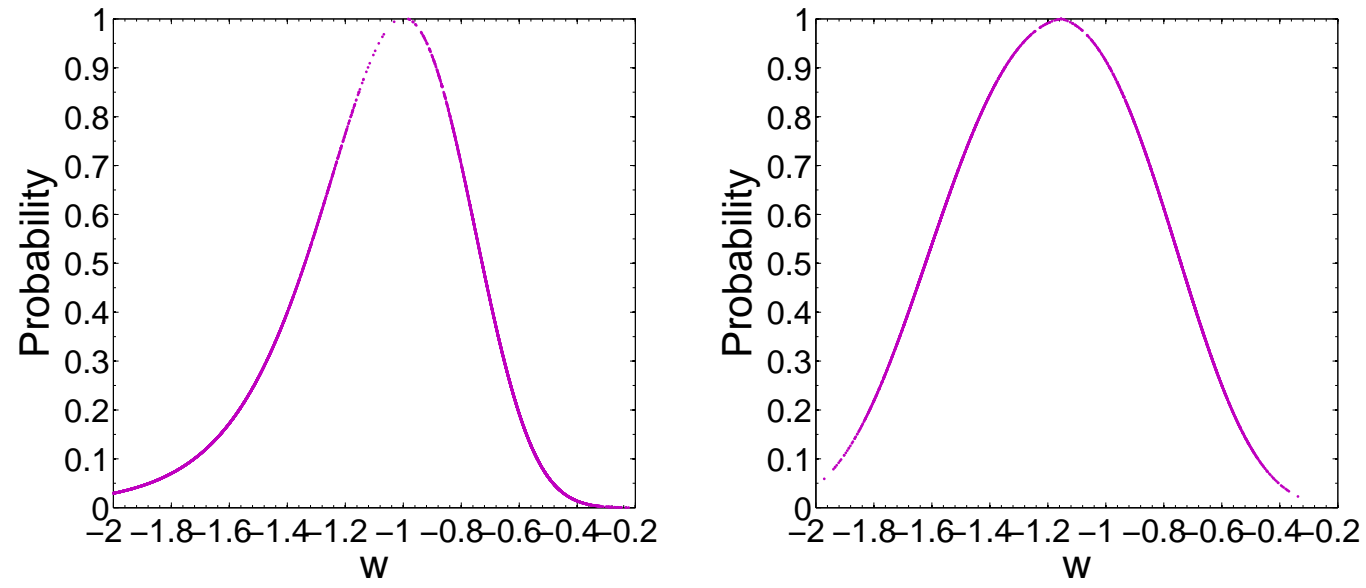

Fig. 4. - The marginalized constraint on $\mathrm{w}$ of $w \mathrm{CDM}$ model from 80 full $D_{d s} / D_{s}$ data and 46 restricted $D_{d s} / D_{s}$ data. 
we marginalize $f_{E}$ through Eq. [6] and perform fits of different cosmological scenarios on the full $n=80$ sample as well as the restricted $n=46$ sample with the results displayed in Table 2,

For the full $n=80$ sample (containing 70 galaxy lenses and 10 strong lensing arcs), first, in $\Lambda$ CDM model where $\Omega_{m}$ is the only free parameter we were able to make a reliable fit on the samples considered. This result is a considerable improvement over Biesiada et al. (2010), where the authors failed to constrain $\Omega_{m}$ with their sample of twenty Einstein rings. Let us compare our results with previously known ones. The current best fit value from cosmological observations is: $\Omega_{\Lambda}=0.73 \pm 0.04$ in the flat case (Davis et al. 2007). Moreover, Komatsu et al. (2009) gave the best-fit parameter $\Omega_{m}=0.274$ for the flat $\Lambda$ CDM model from the WMAP5 results with the BAO and SNe Ia Union data. We find that our value of $\Omega_{m}$ (see Table 2) obtained from the $D_{d s} / D_{s}$ data is consistent with the previous works at $1 \sigma$. Secondly, the best fit for the $w \mathrm{CDM}$ parameter agrees with that inferred from SNe Ia or WMAP 5, and the $\Lambda$ CDM model $(w=-1)$ still falls within the $1 \sigma$ interval from the $D_{d s} / D_{s}$ sample. Hence the agreement is quite good. Thirdly, concerning the evolving equation of state in the CPL parametrization, confidence regions in the $\left(w_{0}, w_{a}\right)$ plane are shown in Fig. [5. One can see that fits for $w_{0}$ and $w_{a}$ are greatly improved as compared with those of Biesiada et al. (2010). The values inferred are also in agreement with the WMAP5 results presented in Hinshaw et al. (2009) including combined WMAP5, BAO and SNe Ia analysis. Moreover, it can be seen that the concordance model $(\Lambda \mathrm{CDM})$ is still included at $1 \sigma$ level for the $D_{d s} / D_{s}$ data applied here. For comparison we also plot the likelihood contours with the Union2 SNe Ia compilation (Amanullah et al. 2010). One can see that the $w$ coefficients obtained from the $D_{d s} / D_{s}$ sample agrees with the respective values derived from supernovae data (almost the whole $2 \sigma$ confidence interval for $w$ from the Union2 data set lies within the $2 \sigma$ CI from the $D_{d s} / D_{s}$ data), which demonstrates the compatibility between the SNe Ia and $D_{d s} / D_{s}$ data. This is also a great improvement over Biesiada et al. (2010), where SNe Ia results and strong lensing results were found marginally inconsistent at $2 \sigma$.

Working on the restricted $n=46$ sample (containing 36 two-image lenses and 10 strong lensing arcs), despite the sample size has decreased dramatically, we find that fits on $\Omega_{m}$ in $\Lambda \mathrm{CDM}$ model are consistent with the standard knowledge (see Fig. 3i) and the best fit for the $w$ parameter in quintessence scenario is higher than inferred from SNe Ia or WMAP5 (see Fig. (4). Moreover, for the fits on $w_{0}$ and $w_{a}$ in CPL parametrization, even though confidence regions get larger in Fig. 5 , the result also turns out to agree with SNe Ia fits. One should also note, that a systematic shift downwards in the $\left(w_{0}, w_{a}\right)$ plane persists. Such a shift in best-fitting parameters inferred from supernovae (standard candles, sensitive to luminosity distance) and BAO (standard rulers, sensitive to angular diameter distance) has already been noticed and discussed in Linder \& Roberts (2008); Biesiada et al. (2010). Our 
result suggests the need for taking a closer look at the compatibility of results derived by using angular diameter distances and luminosity distances, respectively. Recent discussions on the ideas of testing the Etherington reciprocity relation between these two distances can be found in Bassett \& Kunz (2004); Uzan et al. (2004); Holanda, Lima \& Ribeiro (2010); Cao \& Zhu (2011b); Piórkowska, Biesiada \& Maled (2010).

In conclusion our results demonstrate that the method extensively investigated in Biesiada (2006); Grillo et al. (2008); Biesiada et al. (2010); Yu \& Zhu (2010) on simulated and observational data can practically be used to constrain cosmological models. Moreover, good quality measurements of the relevant observational qualities such as the velocity dispersion and Einstein radius turn out to be crucial. Finally, four important effects, neglected here, should be mentioned. One is that both the Einstein rings and X-ray observations of our new lensing sample come from different surveys or satellites (SLACS, LSD and SBAS and Chandra, ROSAT and ASCA, respectively), the differences in detectors and observing strategies may cause systematical errors which are hard to estimate. The second is that the observed image separation is affected by secondary lenses (satellites, nearby galaxies, groups, etc) in many cases. In this case, those lenses should not be used or the true $\theta_{E}$ corresponding to $\sigma_{0}$ should be estimated through realistic modelling. However, most of our samples come from the SLACS survey where the role of environment has been assessed in Treu et al. (2009). Namely, it was found that for SLACS lenses the typical contribution from external mass distribution is no more than a few percent. The third important effect is, that the statistical procedure for cluster lenses relies on many simplifying assumptions. The realistic errors should be estimated by more realistic model of galaxy clusters besides the hydrostatic isothermal spherical symmetric $\beta$-model.The last one is the influence of lineof-sight mass contamination, with the significant effect of the large-scale structure on strong lensing (Bar-Kana 1996; Keeton et al. 1997). More recent results on this issue can be found in Dalal et al. (2005); Momcheva et al. (2006). In this paper, large scale structure effects which change the typical separation between images are also included in the parameter $f_{E}$ an increase of an arbitrary order $f_{E}^{1 / 2}$ in the velocity dispersion is equivalent to an increase of $f_{E}$ in the typical separation $\theta$ (i.e., $\theta \propto \sigma^{2}$ ) (Martel et al. 2002). In order to be complete with the discussion of possible errors one should also notice that the redshifts $z_{s}$ and $z_{l}$ are also known with some accuracy $\delta z_{s}$ and $\delta z_{l}$ which propagates into theoretical distance ratio calculations. In principle one should have accounted for them by suitable numerical simulations. However, based on the experience gained on SNIa (Perlmutter et al. 1999), this effect is likely to be much smaller than systematic errors discussed above. Another straightforward solution based on Poissonian statistics suggests that a sample size of order of a few hundred lenses might reduce the line-of-sight 'noise' contamination down to a few percent (Kubo et al. 2010). However, our $D_{d s} / D_{s}$ data set is really small, and its range of redshift is 
also limited. Fortunately, with the ongoing of various systematic gravitational surveys and more giant arc survey projects carried out by the International X-ray Observatory (IXO) (White et al. 2010), extended Roentgen Survey with an Imaging Telescope Array (eRosita) (Predehl et al. 2010) and the Wide Field X-ray Telescope (WFXT) (Murray \& WFXT Team 2010) being under way, the sample of strong lenses is growing rapidly, which may ease the problem of line-of-sight contamination. Future observations will definitely enlarge our set and make the method applied in this paper more powerful.

\section{Acknowledgments}

This work was supported by the National Natural Science Foundation of China under the Distinguished Young Scholar Grant 10825313 and Grant 11073005, the Ministry of Science and Technology national basic science Program (Project 973) under Grant No. 2012CB821804, the Fundamental Research Funds for the Central Universities and Scientific Research Foundation of Beijing Normal University, and the Polish Ministry of Science Grant No. N N203 390034.

\section{REFERENCES}

Adelman-McCarthy, J., et al. 2007, ApJS, 172, 634

Adelman-McCarthy, J., et al. 2008, ApJS, 175, 297

Allam, S. S., et al. 2004, AJ, 127, 1883

Allam, S. S., et al. 2007, ApJ, 662, L51

Allen, S. W., et al. 2008, MNRAS, 383, 879

Amanullah, R., et al. 2010, ApJ, 716, 712 arXiv:1004.1711

Amati, L., et al. 2008, MNRAS, 391, 577

Bar-Kana, R. 1996, ApJ, 468, 17

Bassett, B. A., \& Kunz, M. 2004, PRD, 69, 101305

Biesiada, M.,Godlowski, W., \& Szydlowski, M. 2005, ApJ, 622, 28

Biesiada, M. 2006, PRD, 73, 023006 
Biesiada, M., Piórkowska, A., \& Malec, B. 2010, MNRAS, 406, 1055

Biesiada, M., Malec, B., \& Piórkowska, A. 2011, RAA, in print

Bonamente et al. 2006, ApJ, 647, 25

Borgani, S., Rosati, P., Tozzi, P., \& Norman, C. 1999, ApJ, 517, 40

Breimer, T. G., \& Sanders, R. H. 1992, MNRAS, 257, 97

Browne, I. W. A., et al. 2003, MNRAS, 341, 13

Burles, S., Nollett, K. M., \& Turner, M. S. 2001, ApJL, 552, L1

Caldwell, R. R., Dave, R., \& Steinhardt, P. J. 1998, PRL, 80, 1582

Caldwell, R. R. 2002, PLB, 545, 23

Cao, S., \& Zhu, Z.-H. 2011, China Series G, 54, 12 [arXiv:1102.2750]

Cao, S., \& Zhu, Z.-H. 2011, A\&A, in press, arXiv:1105.6182

Cavaliere, A., \& Fusco-Femiano, R. 1976, A\&A, 49, 137

Chae, K.-H. 2003, MNRAS, 346, 746

Chae, K.-H., \& Mao, S. D. 2003, ApJ, 599, L61

Chae, K.-H., Chen, G., Ratra, B., \& Lee, D.-W. 2004, ApJ, 607, L71

Chevalier, M., \& Polarski, D. 2001, IJMPD, 10, 213

Christlein, D. 2000, ApJ, 545, 145

Covone, G., et al. 2005, Submitted to A\&A [arXiv:0511332]

Dalal, N., Hennavi, J. F., \& Bode, P. 2005, ApJ, 622, 99

Daly, R. A., et al. 2009, ApJ, 691, 1058

Davis, T. M. et al. 2007, ApJ, 666, 716

Di Pietro, E., \& Claeskens, J. F. 2003, MNRAS, 341, 1299

Dvali, G., Gabadadze, G., \& Porrati, M. 2000, PLB, 485, 208

Eisenstein, D. J., et al. 2005, ApJ, 633, 560 
Fassnacht, \& Cohen, 1998, MNRAS, submitted

Feng, B., Wang, X., \& Zhang, X. 2005, PLB, 607, 35

Freese, K., \& Lewis, M. 2002, PLB, 540, 1

Gladders, M. D., et al. 2003, ApJ, 593, 48

Grillo, C., Lombardi, M., \& Bertin, G. 2008, A\&A, 477, 397

Hennawi, J. F., et al. 2008, AJ, 135, 664

Hinshaw, G., et al. 2009, ApJS, 180, 225

Holanda, R. F. L., Lima, J. A. S., \& Ribeiro, M. B., 2010, ApJ, 722, L233

Itoh, N., Kohyama, Y., \& Nozawa, S. 1998, ApJ, 502, 7

Jin, K.-J., Zhang, Y.-Z., \& Zhu, Z.-H. 2000, PLA, 264,335

Jones, M. E., et al. 2005, MNRAS, 357, 518

Kamenshchik, A., Moschella, U., \& Pasquier, V. 2001, PLB, 511, 265

Keeton, C. R., Kochanek, C. S., \& Seljak, U. 1997, ApJ, 482, 604

Keeton, C. R., Christlein, D., \& Zabludoff, A. I. 2000, ApJ, 545, 129

Keeton, C. R. 2001, ApJ, 561, 46

King, L. J., et al. 1997, MNRAS, 289, 450

Kochanek, C. S., 1992, ApJ, 384, 1

Kochanek, C. S. 1996, ApJ, 466, 638

Kochanek, C. S., \& White, M. 2001, ApJ, 559, 531

Komatsu, E., et al. [WMAP Collaboration], 2009, AJS, 180, 330

Koopmans, L. V. E., \& Treu, T. 2002, ApJ, 568, L5

Koopmans, L. V. E., \& Treu, T. 2003, ApJ, 583, 606

Koopmans, L. V. E., et al. 2006, ApJ, 649, 599

Koopmans, L. V. E., et al. 2009, ApJ, 703, L51 
Kowalski, M., et al. 2008, ApJ, 686, 749

Kubo, J. M., et al. 2010, accepted by ApJL, arXiv:1010.3037v2

Linder, E. V. 2003, PRD, 68, 083503

Linder, E. V., \& Roberts, G. 2008, J. Cosmol. Astropart. Phys., 0806, 004

Lynds, R., \& Petrosian, V. 1986, AAS, 18, 1014

Mao, S. D., \& Schneider, P. 1998, MNRAS, 295, 587

Martel, H., Premadi, P., \& Matzner, R. 2002, ApJ, 570, 17

Mitchell, J. L., Keeton, C. R., Frieman, J. A., \& Sheth, R. K. 2005, ApJ, 622, 81

Momcheva, I., Williams, K., Keeton, C. R., \& Zabludoff, A. 2006, ApJ, 641, 169

Murray, S. S., \& WFXT Team. 2010, AAS, 41, 520

Narayan, R. \& Bartelmann, M. 1996, In: "Lectures on Gravitational Lensing", arXiv:9606001

Newton, E., et al. 2011, accepted by ApJ, arXiv:1104.2608

Nozawa, S., Itoh, N., \& Kohyama, Y. 1998, ApJ, 508, 17

Nozawa, S., Itoh, N., Suda, Y., \& Ohhata, Y. 2006, Nuovo Cimento, 121 B, 487

Ofek, E. O., Rix, H.-W., \& Maoz, D. 2003, MNRAS, 343, 639

Ono, T., Masai, K., \& Sasaki, S. 1999, PASJ, 51, 91

Ota, N., \& Mitsuda, K. 2004, A\&A, 428, 757

Paczynski, B., \& Gorski, K. 1981, ApJL, 248, L101

Perlmutter, S., et al. 1999, ApJ, 517, 565

Piórkowska, A., Biesiada, M. \& Malec, B., 2011, Acta Phys. Polon. B, 42, 2297

Predehl, P. et al. 2010, arXiv:1001.2502

Press, W. H., \& Schechter, P. 1974, ApJ, 187, 425

Reese, E. D., et al. 2002, ApJ, 581, 53

Richard, J., et al. 2007, ApJ, 662, 781; Abell 68 
Riess, A. G., et al. 1998, AJ, 116, 1009

Riess, A. G., et al. 2004, ApJ, 607, 665

Rosati, P., Borgani, S., \& Norman, C. 2002, ARA\&A, 40, 539

Rusin, D., \& Kochanek, C. S. 2005, ApJ, 623, 666

Samushia, L., \& Ratra, B. 2008, ApJ, 680, L1

Schmidt, R. W., Allen, S. W., \& Fabian, A. C. 2004, MNRAS, 352, 1413

Schneider, P., Ehlers, J., \& Falco, E. E. 1992, Gravitational Lenses

Sereno, M. 2002, A\&A, 393, 757

Sereno, M., \& Longo, G. 2004, MNRAS, 354, 1255

Sheth, R. K., et al. 2003, ApJ, 594, 225

Spergel, D. N. et al. 2007, ApJS, 170, 377

Stoughton, C., et al. 2002, AJ, 123, 485

Sunyaev, R. A., \& Zeldovich, Y. B. 1972, Comments on Astrophysics and Space Physics, 4, 173

Treu, T., \& Koopmans, L. V. E. 2004, ApJ, 611, 739

Treu, T., et al. 2006a, ApJ, 640, 662

Treu, T., et al. 2006b, ApJ, 650, 1219

Treu, T., et al. 2009, ApJ, 690, 670

Uzan, J. P., Aghanim, N., \& Mellier, Y. 2004, PRD, 70, 083533

Walsh, D., Carswell, R. F., \& Weymann, R. J. 1979, Nature, 279, 381

Weinberg, S. 1989, Rev. Mod. Phys., 61, 1

White, R. E. \& Davis, D. S. 1996, American Astronomical Society Meeting, 28, 1323

White, N. E., et al. 2010, arXiv:1001.2843

Yu, H., \& Zhu, Z.-H., accepted by RAA, arXiv:1011.6060 
Zhang, H., \& Zhu, Z.-H. 2006, PRD, 73, 043518

Zhu, Z.-H., \& Wu, X.-P. 1997, A\&A, 324, 483

Zhu, Z.-H. 2000, MPLA, 15, 1023

Zhu, Z.-H. 2000, IJMPD, 9, 591

Zhu, Z.-H. 2004, A\&A, 423, 421

Zhu, Z.-H., \& Fujimoto, M. 2004, ApJ, 602, 12

Zhu, Z.-H., Fujimoto, M., \& He, X. 2004, ApJ, 603, 365

Zhu, Z.-H., \& Mauro, S. 2008, A\&A, 487, 831

Zhu, Z.-H., et al. 2008, A\&A, 483, 15 
Table 1: Values of $\mathcal{D}=D_{d s} / D_{s}$ from lensing galaxy clusters and combined SLACS+LSD lens samples. The two-image lenses are written in bold.

\begin{tabular}{|c|c|c|c|c|c|}
\hline System & $\mathrm{z}_{d}$ & $\mathrm{z}_{s}$ & $\mathcal{D}^{\text {obs }}$ & $\sigma_{\mathcal{D}}$ & ref \\
\hline MS 0451.6-0305 & 0.550 & 2.91 & 0.785 & 0.087 & Bonamente et al. (2006); Yu \& Zhu (2010) \\
\hline $3 \mathrm{C} 220.1$ & 0.61 & 1.49 & 0.611 & 0.530 & Ota \& Mitsuda (2004); Yu \& Zhu (2010) \\
\hline CL0024.0 & 0.391 & 1.675 & 0.919 & 0.430 & Ota \& Mitsuda (2004); Yu \& Zhu (2010) \\
\hline Abell 2390 & 0.228 & 4.05 & 0.737 & 0.053 & Ota \& Mitsuda (2004); Yu \& Zhu (2010) \\
\hline Abell 2667 & 0.226 & 1.034 & 0.837 & 0.124 & Ota \& Mitsuda (2004); Covone et al. (2005); Yu \& Zhu (2010) \\
\hline Abell 68 & 0.255 & 1.6 & 0.982 & 0.225 & Bonamente et al. (2006); Richard et al. (2007); Yu \& Zhu (2010) \\
\hline MS 1512.4 & 0.372 & 2.72 & 0.734 & 0.330 & Ota \& Mitsuda (2004); Yu \& Zhu (2010) \\
\hline MS 2137.3-2353 & 0.313 & 1.501 & 0.778 & 0.105 & Ota \& Mitsuda (2004); Yu \& Zhu (2010) \\
\hline MS 2053.7 & 0.583 & 3.146 & 0.968 & 0.209 & Ota \& Mitsuda (2004); Bonamente et al. (2006) \\
\hline PKS 0745-191 & 0.103 & 0.433 & 0.818 & 0.065 & Ota \& Mitsuda (2004); Yu \& Zhu (2010) \\
\hline SDSS J0037-0942 & 0.1955 & 0.6322 & 0.6825 & 0.1026 & Biesiada et al. (2010) \\
\hline SDSS J0216-0813 & 0.3317 & 0.5235 & 0.3632 & 0.0684 & Biesiada et al. (2010) \\
\hline SDSS J0737+3216 & 0.3223 & 0.5812 & 0.3039 & 0.0458 & Biesiada et al. (2010) \\
\hline SDSS J0912+0029 & 0.1642 & 0.324 & 0.5325 & 0.0789 & Biesiada et al. (2010) \\
\hline SDSS J0956+5100 & 0.2405 & 0.47 & 0.414 & 0.0628 & Biesiada et al. (2010) \\
\hline SDSS J0959+0410 & 0.126 & 0.5349 & 0.5599 & 0.1152 & Biesiada et al. (2010) \\
\hline SDSS J1250+0523 & 0.2318 & 0.795 & 0.6179 & 0.0996 & Biesiada et al. (2010) \\
\hline SDSS J1330-0148 & 0.0808 & 0.7115 & 0.7762 & 0.1184 & Biesiada et al. (2010) \\
\hline SDSS J1402+6321 & 0.2046 & 0.4814 & 0.6575 & 0.1166 & Biesiada et al. (2010) \\
\hline SDSS J1420+6019 & 0.0629 & 0.5352 & 0.8593 & 0.1268 & Biesiada et al. (2010) \\
\hline SDSS J1627-0053 & 0.2076 & 0.5241 & 0.5078 & 0.0779 & Biesiada et al. (2010) \\
\hline SDSS J1630+4520 & 0.2479 & 0.7933 & 0.8114 & 0.1347 & Biesiada et al. (2010) \\
\hline SDSS J2300+0022 & 0.2285 & 0.4635 & 0.5531 & 0.0951 & Biesiada et al. (2010) \\
\hline SDSS J2303+1422 & 0.1553 & 0.517 & 0.8651 & 0.1519 & Biesiada et al. (2010) \\
\hline SDSS J2321-0939 & 0.0819 & 0.5324 & 0.896 & 0.1312 & Biesiada et al. (2010) \\
\hline Q0047-2808 & 0.485 & 3.595 & 0.8872 & 0.1606 & Biesiada et al. (2010) \\
\hline CFRS03-1077 & 0.938 & 2.941 & 0.6834 & 0.1377 & Biesiada et al. (2010) \\
\hline HST 14176 & 0.81 & 3.399 & 0.9757 & 0.1795 & Biesiada et al. (2010) \\
\hline HST 15433 & 0.497 & 2.092 & 0.929 & 0.2067 & Biesiada et al. (2010) \\
\hline MG 2016 & 1.004 & 3.263 & 0.5035 & 0.1234 & Biesiada et al. (2010) \\
\hline SDSS J0029-0055 & 0.227 & 0.9313 & 0.6356 & 0.1317 & Newton et al. (2011) \\
\hline SDSS J0044+0113 & 0.1196 & 0.1965 & 0.3877 & 0.0573 & Newton et al. (2011) \\
\hline SDSS J0109+1500 & 0.2939 & 0.5248 & 0.3803 & 0.0766 & Newton et al. (2011) \\
\hline SDSS J0252+0039 & 0.2803 & 0.9818 & 1.3426 & 0.2636 & Newton et al. (2011) \\
\hline SDSS J0330-0020 & 0.3507 & 1.0709 & 0.8498 & 0.2109 & Newton et al. (2011) \\
\hline SDSS J0405-0455 & 0.0753 & 0.8098 & 1.0851 & 0.1628 & Newton et al. (2011) \\
\hline SDSS J0728+3835 & 0.2058 & 0.6877 & 0.9477 & 0.1448 & Newton et al. (2011) \\
\hline SDSS J0822+2652 & 0.2414 & 0.5941 & 0.6056 & 0.1004 & Newton et al. (2011) \\
\hline SDSS J0841+3824 & 0.1159 & 0.6567 & 0.9671 & 0.143 & Newton et al. (2011) \\
\hline SDSS J0935-0003 & 0.3475 & 0.467 & 0.1926 & 0.0437 & Newton et al. (2011) \\
\hline SDSS J0936+0913 & 0.1897 & 0.588 & 0.6409 & 0.0953 & Newton et al. (2011) \\
\hline SDSS J0946+1006 & 0.2219 & 0.6085 & 0.6927 & 0.1452 & Newton et al. (2011) \\
\hline SDSS J0955+0101 & 0.1109 & 0.3159 & 0.8571 & 0.159 & Newton et al. (2011) \\
\hline SDSS J0959+4416 & 0.2369 & 0.5315 & 0.5599 & 0.1152 & Newton et al. (2011) \\
\hline SDSS J1016+3859 & 0.1679 & 0.4394 & 0.6204 & 0.0963 & Newton et al. (2011) \\
\hline SDSS J1020+1122 & 0.2822 & 0.553 & 0.524 & 0.0931 & Newton et al. (2011) \\
\hline SDSS J1023+4230 & 0.1912 & 0.696 & 0.836 & 0.1454 & Newton et al. (2011) \\
\hline SDSS J1029+0420 & 0.1045 & 0.6154 & 0.7952 & 0.1231 & Newton et al. (2011) \\
\hline SDSS J1032+5322 & 0.1334 & 0.329 & 0.4082 & 0.0618 & Newton et al. (2011) \\
\hline SDSS J1103+5322 & 0.1582 & 0.7353 & 0.9219 & 0.159 & Newton et al. (2011) \\
\hline SDSS J1106+5228 & 0.0955 & 0.4069 & 0.6222 & 0.0928 & Newton et al. (2011) \\
\hline SDSS J1112+0826 & 0.273 & 0.6295 & 0.5052 & 0.0885 & Newton et al. (2011) \\
\hline SDSS J1134+6027 & 0.1528 & 0.4742 & 0.6687 & 0.1005 & Newton et al. (2011) \\
\hline SDSS J1142+1001 & 0.2218 & 0.5039 & 0.6967 & 0.1735 & Newton et al. (2011) \\
\hline SDSS J1143-0144 & 0.106 & 0.4019 & 0.8061 & 0.1182 & Newton et al. (2011) \\
\hline SDSS J1153+4612 & 0.1797 & 0.8751 & 0.7138 & 0.1305 & Newton et al. (2011) \\
\hline SDSS J1204+0358 & 0.1644 & 0.6307 & 0.6381 & 0.1132 & Newton et al. (2011) \\
\hline SDSS J1205+4910 & 0.215 & 0.4808 & 0.5365 & 0.0803 & Newton et al. (2011) \\
\hline SDSS J1213+6708 & 0.1229 & 0.6402 & 0.5783 & 0.0883 & Newton et al. (2011) \\
\hline SDSS J1218+0830 & 0.135 & 0.7172 & 1.0498 & 0.158 & Newton et al. (2011) \\
\hline SDSS J1403+0006 & 0.1888 & 0.473 & 0.6352 & 0.1332 & Newton et al. (2011) \\
\hline SDSS J1416+5136 & 0.2987 & 0.8111 & 0.8259 & 0.2134 & Newton et al. (2011) \\
\hline SDSS J1430+4105 & 0.285 & 0.5753 & 0.509 & 0.1266 & Newton et al. (2011) \\
\hline SDSS J1432+6317 & 0.123 & 0.6643 & 1.1048 & 0.1662 & Newton et al. (2011) \\
\hline SDSS J1436-0000 & 0.2852 & 0.8049 & 0.775 & 0.1563 & Newton et al. (2011) \\
\hline SDSS J1443+0304 & 0.1338 & 0.4187 & 0.6439 & 0.1 & Newton et al. (2011) \\
\hline SDSS J1451-0239 & 0.1254 & 0.5203 & 0.7262 & 0.1275 & Newton et al. (2011) \\
\hline SDSS J1525+3327 & 0.3583 & 0.7173 & 0.6526 & 0.1611 & Newton et al. (2011) \\
\hline SDSS J1531-0105 & 0.1596 & 0.7439 & 0.7628 & 0.1147 & Newton et al. (2011) \\
\hline SDSS J1538+5817 & 0.1428 & 0.5312 & 0.972 & 0.172 & Newton et al. (2011) \\
\hline SDSS J1621+3931 & 0.2449 & 0.6021 & 0.8042 & 0.1765 & Newton et al. (2011) \\
\hline SDSS J1636+4707 & 0.2282 & 0.6745 & 0.7093 & 0.1276 & Newton et al. (2011) \\
\hline SDSS J2238-0754 & 0.1371 & 0.7126 & 1.1248 & 0.1812 & Newton et al. (2011) \\
\hline SDSS J2341+0000 & 0.186 & 0.807 & 1.1669 & 0.2049 & Newton et al. (2011) \\
\hline $\mathrm{Q} 0957+561$ & 0.36 & 1.41 & 1.3103 & 0.1474 & Newton et al. (2011) \\
\hline PG1115+080 & 0.31 & 1.72 & 0.7036 & 0.1604 & Newton et al. (2011) \\
\hline MG1549+3047 & 0.11 & 1.17 & 0.5728 & 0.1194 & Newton et al. (2011) \\
\hline $\mathrm{Q} 2237+030$ & 0.04 & 1.169 & 0.6685 & 0.22 & Newton et al. (2011) \\
\hline CY2201-3201 & 0.32 & 3.9 & 0.8526 & 0.305 & Newton et al. (2011) \\
\hline $\mathrm{B} 1608+656$ & 0.63 & 1.39 & 0.646 & 0.2154 & Newton et al. (2011) \\
\hline
\end{tabular}


Table 2: Fits to different cosmological models from 80 full $D_{d s} / D_{s}$ data and 46 restricted $D_{d s} / D_{s}$ data. Fixed value of $\Omega_{m}=0.27$ is assumed except $\Lambda$ CDM.

\begin{tabular}{cll}
\hline \hline Cosmological model & Best-fitting parameters $(n=80)$ & Best-fitting parameters $(n=46)$ \\
\hline$\Lambda \mathrm{CDM}$ & $\Omega_{m}=0.20_{-0.07}^{+0.07}$ & $\Omega_{m}=0.26_{-0.10}^{+0.11}$ \\
\hline$w \mathrm{CDM}$ & $w=-1.02_{-0.26}^{+0.26}$ & $w=-1.15_{-0.35}^{+0.34}$ \\
\hline $\mathrm{CPL}$ & $w_{0}=0.60 \pm 1.76$ & $w_{0}=-0.24 \pm 2.42$ \\
& $w_{a}=-7.37 \pm 8.05$ & $w_{a}=-6.35 \pm 9.75$ \\
\hline
\end{tabular}
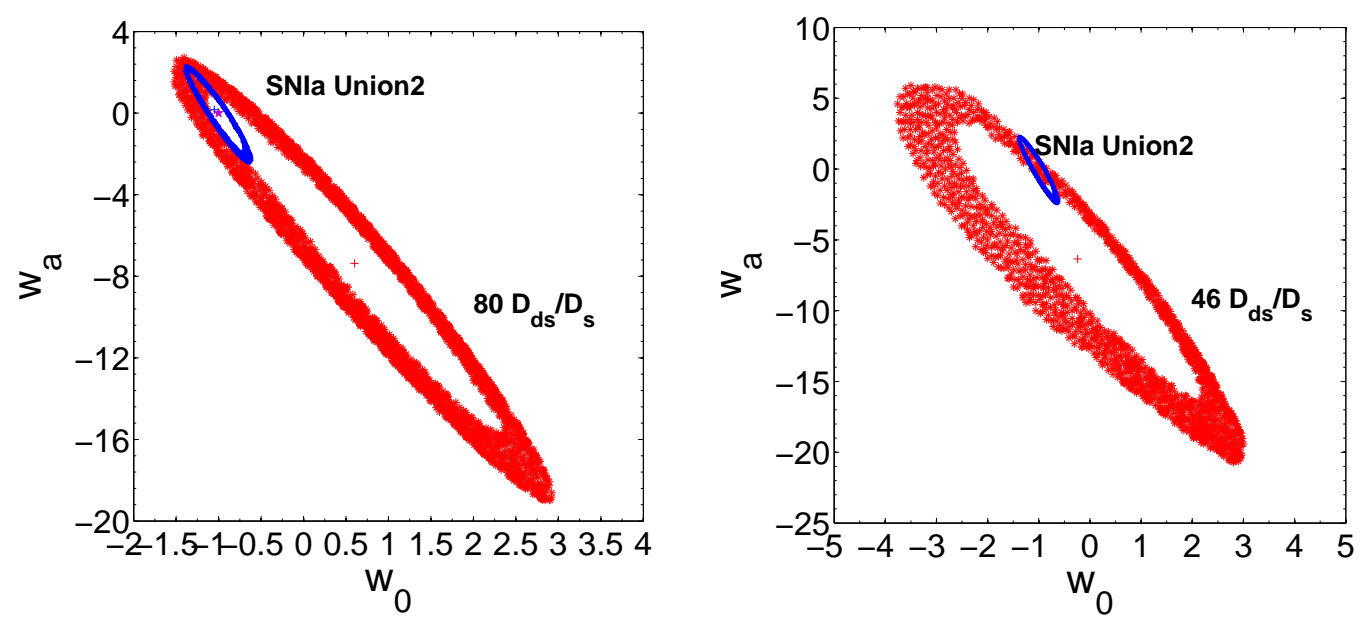

Fig. 5.- The 68.3 and $95.8 \%$ confidence regions for CPL parametrization in the $\left(w_{0}, w_{a}\right)$ plane obtained from 80 full $D_{d s} / D_{s}$ data, 46 restricted $D_{d s} / D_{s}$ data, and 557 Union2 SNe Ia data. The crosses represent the best-fit points and a star corresponding to $\Lambda$ CDM model is also added for reference. 